\title{
Learning interaction control policies by demonstration
}

\author{
Vasiliki Koropouli, Dongheui Lee and Sandra Hirche
}

\begin{abstract}
This paper explores learning of interaction force skills by human demonstration in dynamic interaction tasks. Skillful force regulation is required in many cases to achieve the goal of a task and at the same time, not to cause undesired stress on the manipulator or the object under manipulation which could result in physical failure. For example, manipulation of compliant objects with varying physical properties or artistic tasks such as engraving require skillful force modulation. Humans gracefully manipulate objects by using their sense of touch and skillfully regulating exerted forces. To learn the demonstrated force for a task by demonstration, an interaction force control policy, in terms of a goal-directed dynamical system, is proposed which stems from the parallel force/position control. The control policy is parameterized and its parameters are learned by Locally Weighted Regression from human demonstrated data to learn a force trajectory. Scaling of learned force is possible by modifying the goal of the system. The proposed method is evaluated in virtual manipulation tasks using a two degrees-of-freedom haptic device.
\end{abstract}

\section{INTRODUCTION}

In manipulation tasks, both positional and force skills are needed to successfully interact with the environment. To draw on a rough surface, for example, positional skills are required to counteract disturbances. To control constrained movements, however, skillful force regulation is also of interest. In case of compliant environments, a dynamic interaction is established between the end-effector and the object in contact and control of interaction force is required [4]. The interaction force establishes a relationship between exerted force by the manipulator and change in the kinematic state of the point of contact with the environment.

Looking into biological systems, the motor control mechanism consists of both feedforward and feedback components where the feedforward mechanism allows for fast and smooth movements [5]. Inspired from the motor control mechanism in humans, we are interested in learning feedforward force control skills for robots and combine with feedback control to ensure successful execution in different contexts.

In many robotic tasks, feedforward force control is necessary to guarantee efficiency and safety during task execution. Many environments, for example, are not homogeneous and their stiffness varies along their dimensions. Let us imagine of a soft object which is overlaid by an even softer material for insulation or protection, shown in Fig. 1(a). Humans gracefully manipulate such objects by exerting low forces

The authors are with the Department of Electrical Engineering and Information Technology, Technical University of Munich, D-80290 Munich, Germany. vicky@lsr.ei.tum.de \{dhlee, hirche\}etum.de

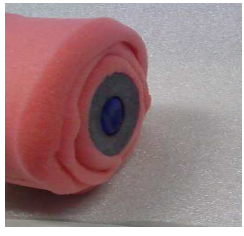

(a)

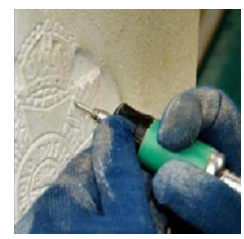

(b)
Fig. 1. (a) A pipe (blue) covered by two different soft materials (grey and pink). (b) Engraving of a soft-plastic material.

at the beginning to press the external soft layer and higher forces afterwards to keep contact with the object and increase stability and manipulability. Exertion of high forces from the beginning could result in instability or wrenching on the object. Force tuning is also required in artistic manipulation tasks such as engraving where shaping outcome depends on how much force the user imposes on a material, see Fig. 1(b).

Based on the necessity of learning force skills for efficient and safe manipulation, in this work, we propose a method to learn force tuning skills by human demonstration to interact with compliant external dynamics.

Most works in imitation learning so far are focused on learning positional skills, see e.g. [3], [6], [7]. In [6], a methodology based on Gaussian Mixture Regression is presented to extract the essential features of a task from multiple demonstrations. In [7], movement primitives are learned by human demonstration which exhibit robustness to perturbations during execution. In [3], an extended approach similar to [7] is proposed to learn free-space movement primitives categorized into discrete and rhythmic control policies. In addition, motion learning by imitation is employed to teach robots how to physically interact with humans where impedance control ensures compliant robot behavior while in contact with the human [8].

In contrast to previous works which focus on learning motion skills by demonstration, in the present work, we propose a method to learn interaction force skills for an endeffector in contact with a compliant environment. To this aim, we first model an interaction force by a nominal interaction control policy expressed by the parallel force/position control law [1]. We transform this policy into a goal-driven dynamical system which evolves in phase space. We add to the system a parameterized function to compensate for the uncertainty between desired and modeled task force. This parametric function is learned by locally weighted regression from human demonstrated force and position data [3]. By doing so, we achieve fitting of the nominal force model to a desired force signal. Scaling of learned force is possible, by modifying the goal of the system, to generalize learned 


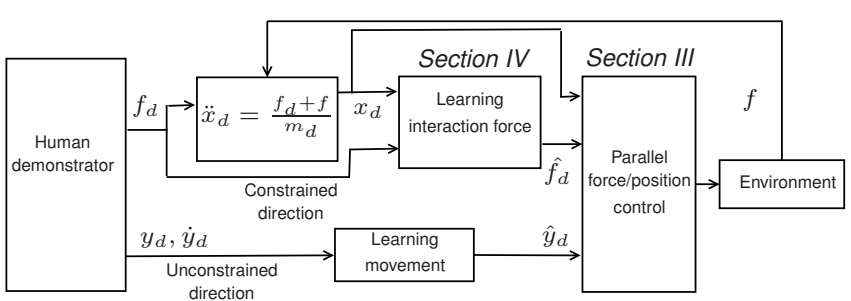

Fig. 2. Schematic overview of the proposed method. Both user force $f_{d}$ and environmental force $f$ are exerted on the handle which moves accordingly.

skills to new contexts. The proposed method is evaluated in virtual manipulation tasks by using a two degrees-of-freedom (DOF) haptic device. A schematic overview of the proposed method is presented in Fig. 2.

This paper is organized as follows. In Section II, we present the problem setting and the main steps of our approach. Section III describes the parallel force/position control and Section IV introduces our proposed method of learning interaction forces by demonstration. Finally, in Section $\mathrm{V}$, we evaluate our method in a virtual manipulation scenario.

\section{Problem Setting}

In the present work, we propose a method to learn interaction force skills by demonstration to physically interact with compliant dynamics. In compliant environments, a dynamic interaction is established between the end-effector and the environment, and the characteristics of this interaction depend upon the human intention/task and the physical properties of the object in contact, given a manipulator with known properties. Based on that, we are interested in learning a model for an interaction force which considers the endeffector, not as an isolated system, but physically coupled with its environment.

To learn an interaction force demonstrated by the human user, we take the following steps. First, we model a force trajectory in terms of an interaction control policy represented by the parallel force/position control law [1]. In this policy, we represent the nominal environmental dynamics by a linear spring. By expressing the policy as a goal-driven dynamical system, we achieve representation of a time-varying force signal by time-invariant dynamics. Second, we add to the system a parametric function to account for the deviation from the true environmental and task dynamics. Third, we learn this parametric function from human demonstrated force $f_{d}$ and position $x_{d}$ data to allow for fitting of the system's dynamics to the signal $f_{d}$. The proposed dynamical system also exhibits spatial invariance which allows for force scaling by modifying the goal of the system. This property is exploited in the experiments to manipulate objects with different physical properties, e.g. generalize from a soft to a stiffer environment.

In this paper we consider a parallel force/position control law as nominal control law as it is a direct force control scheme which allows for explicit force input and takes into account the dynamic coupling between manipulator and environment.

\section{PARALLEL FORCE/POSITION CONTROL}

In this section, we describe the parallel force/position control scheme presented in [1]. This control scheme allows for parallel control of force and position of a manipulator in full-dimensional task space without the use of selection matrices as in hybrid control. Force control is prioritized over position control in the constrained directions of movement through appropriate position and force feedback control actions. Due to its special design, the controller can deal with inconsistencies between planning and actual geometry of the task where, in this case, it is not known a priori which are the constrained and unconstrained directions of movement.

When an end-effector is in contact with the environment, its equation of motion in the operational space is given by

$$
M(x) \ddot{x}+C(x, \dot{x})+G(x)+f=f_{m},
$$

where $\boldsymbol{x}, \dot{\boldsymbol{x}}$ and $\ddot{\boldsymbol{x}}$ are $n \times 1$ vectors representing endeffector's position, velocity and acceleration respectively and $n$ is the operational space dimension. The term $M$ is a symmetric and positive semidefinite pseudo-inertia matrix, $C$ Coriolis and centrifugal forces, $G$ the gravity force, $f_{m}$ a vector of generalized forces and $f$ the force that the manipulator exerts onto the environment. The term $M$ is an $n \times n$ matrix and $\boldsymbol{C}, \boldsymbol{G}, \boldsymbol{f}_{\boldsymbol{m}}$ and $\boldsymbol{f}$ are $n \times 1$ vectors. Note that end-effector dynamics (1) is nonlinear due to inertial coupling, Coriolis and centrifugal forces.

We assume that the environment is stiff, elastically compliant and frictionless. Since planar surfaces have been successful in approximation of surfaces of regular curvature [1], environmental surface is considered locally planar at the contact points for reasons of simplicity. By compensating for nonlinearities and decoupling dynamics, the end-effector becomes equivalent to an inertia moving with acceleration $\ddot{x}$, see [1], [10]. Considering the position and force control $\boldsymbol{u}(\boldsymbol{x}, \boldsymbol{f})$, the end-effector's equation of motion becomes

$$
M_{d} \ddot{x}=M_{d} \ddot{x}_{d}+u(x, f),
$$

where $\boldsymbol{M}_{d}$ is an $n \times n$ inertia matrix and $\ddot{x}_{d}$ the desired acceleration vector. Equation (2) constitutes an acceleration control law where the control action $u$ is defined by

$$
\boldsymbol{u}(\boldsymbol{x}, \boldsymbol{f})=\boldsymbol{u}_{p}(\boldsymbol{x})+\boldsymbol{u}_{f}(\boldsymbol{f}),
$$

with the terms $u_{p}$ and $u_{f}$ being the feedback components of the position and the force error respectively. Position control is realized based on a resolved acceleration control scheme [1], [2]

$$
\boldsymbol{u}_{p}=\boldsymbol{K}_{d} \dot{\boldsymbol{e}}_{p}+\boldsymbol{K}_{p} \boldsymbol{e}_{p}
$$

The terms $\boldsymbol{K}_{d}$ and $\boldsymbol{K}_{p}$ are $n \times n$ gain matrices and $\boldsymbol{e}_{p}$ is the position error defined by $\boldsymbol{e}_{p}=\boldsymbol{x}_{\boldsymbol{d}}-\boldsymbol{x}$. The term $\dot{\boldsymbol{e}}_{p}$ represents the derivative of the position error and $x_{d}$ is the vector of desired end-effector position. In the constrained directions of movement, force control is desired and thus force should prevail over position control. To achieve that, 
a proportional-integral control action is taken on the force error

$$
\boldsymbol{u}_{f}=\boldsymbol{K}_{f} \boldsymbol{e}_{f}+\boldsymbol{K}_{i} \int_{0}^{t} \boldsymbol{e}_{f} d \tau
$$

where $e_{f}=f_{d}-f$ is the force error and $f_{d}$ the desired contact force which is an $n \times 1$ vector. The force control action prevails over the position control action (4) in presence of non-zero contact force error [1]. The terms $\boldsymbol{K}_{f}$ and $\boldsymbol{K}_{i}$ are $n \times n$ control gain matrices. The combination of (2)-(5) yields

$$
\boldsymbol{M}_{d} \ddot{\boldsymbol{e}}_{p}+\boldsymbol{K}_{d} \dot{\boldsymbol{e}}_{p}+\boldsymbol{K}_{p} \boldsymbol{e}_{p}+\boldsymbol{K}_{f} \boldsymbol{e}_{f}+\boldsymbol{K}_{i} \int_{0}^{t} \boldsymbol{e}_{f} d \tau=0 .
$$

For dynamic decoupling in operational space, gain matrices $\boldsymbol{M}_{d}, \boldsymbol{K}_{d}, \boldsymbol{K}_{p}, \boldsymbol{K}_{f}$ and $\boldsymbol{K}_{i}$ are defined diagonal. Elements along the diagonal do not need to be the same. Here, for the ease of notation we choose equal gains in all directions, i.e. $m_{d}, k_{d}, k_{p}, k_{f}$ and $k_{i}$ represent the elements of the matrices $\boldsymbol{M}_{d}, \boldsymbol{K}_{d}, \boldsymbol{K}_{p}, \boldsymbol{K}_{f}$ and $\boldsymbol{K}_{i}$, respectively.

For simplicity we consider the end-effector position in 2dimensional space where $x$ denotes the constrained and $y$ the unconstrained direction of movement. Similarly, the desired end-effector position in these directions is represented by $x_{d}$ and $y_{d}$. Movement in the direction normal to the environmental plane is constrained while in the other direction, movement is unconstrained since friction forces are neglected and the dynamics of the task decoupled by assumption. As nominal environment model we consider a linear timeinvariant spring law [1], [9], i.e. the nominal interaction force in the direction normal to the environmental plane is given by

$$
f=k x,
$$

where we assume the origin of coordinate system at the rest position of the plane and $k$ the nominal stiffness coefficient of the material in contact. By substituting $e_{p}$ and $e_{f}$ into (6) and decoupling into constrained and unconstrained direction of movement, we can write

$$
\begin{aligned}
& m_{d} \ddot{x}+k_{d} \dot{x}+\left(k_{p}+k k_{f}\right) x+k k_{i} \int_{0}^{t} x d \tau= \\
& m_{d} \ddot{x}_{d}+k_{d} \dot{x}_{d}+k_{p} x_{d}+k_{f} f_{d}+k_{i} \int_{0}^{t} f_{d} d \tau,
\end{aligned}
$$

for the constrained direction of movement and

$$
m_{d} \ddot{y}+k_{d} \dot{y}+k_{p} y=m_{d} \ddot{y}_{d}+k_{d} \dot{y}_{d}+k_{p} y_{d},
$$

for the unconstrained direction. Equation (8) can be simplified to

$$
\begin{aligned}
& m_{d} \ddot{x}+k_{d} \dot{x}+\left(k_{p}+k k_{f}\right) x+k k_{i} \int_{0}^{t} x d \tau= \\
& k_{p} x_{d}+k_{f} f_{d}+k_{i} \int_{0}^{t} f_{d} d \tau,
\end{aligned}
$$

when the aim is to achieve a position setpoint $x_{d}$, see [1] for more details. It also holds as approximation for low desired accelerations and velocities.

\section{LEARNING BY DEMONSTRATION}

In this section, we propose an approach to learn interaction forces by demonstration by learning a parameterized interaction control policy. First, we encode the interaction force in terms of a nominal control policy which stems from the parallel force/position control law described in Section III and we represent this policy by a goal-directed dynamical system. To compensate for the deviation between true task force and force estimated by the nominal policy, we add a parametric function to the dynamical system and learn that from demonstrated data to allow for fitting of the system to demonstrated force.

\section{A. A nominal force representation}

We represent the force control law (10) by a time-invariant dynamical system. In this section the more challenging part of constrained movement is considered, i.e. only the direction normal to the environmental plane where a contact force exists. For learning the unconstrained motion the approach from [3] can be employed. We model the nominal interaction force by $\hat{f}_{d}$ which is encoded in terms of the following dynamical system of three states $\left(\int x, x, z\right)$

$$
\begin{gathered}
\dot{x}=z-x-\gamma_{z} \int x, \quad \dot{z}=\alpha_{z}\left(\beta_{z}\left(g^{\prime}-x\right)-z\right), \\
\hat{f}_{d}=k x
\end{gathered}
$$

where $k$ represents the nominal stiffness of the environment. The term $g^{\prime}$ represents the goal of the system and $\alpha_{z}, \beta_{z}$, $\gamma_{z}$ and $k$ are parameters. Note that representation of the parallel position-force control law is inspired from the dynamical system representation for position control proposed in [3] and is derived through a partial state transformation $z=\dot{x}+x+\gamma_{z} \int_{0}^{t} x d \tau$. Accordingly, the goal trajectory is defined by

$$
g^{\prime}=\frac{c}{\alpha_{z} \beta_{z}} .
$$

where $c=m_{d}^{-1}\left(k_{p} x_{d}+k_{f} f_{d}+k_{i} \int_{0}^{t} f_{d} d \tau\right)$ and

$\alpha_{z}=\frac{k_{d}}{m_{d}}-1, \beta_{z}=\frac{k_{p}+k k_{f}-\gamma_{z}}{m_{d} \alpha_{z}}-1, \gamma_{z}=\frac{k k_{i}}{m_{d} \alpha_{z}}$.

The terms in (13)-(14) are defined such that (11) is equivalent to (10). The system (11), (12) receives as input demonstrated position $x_{d}$ and force $f_{d}$ data, and outputs an estimate for the force $\hat{f}_{d}$.

The dynamics (11)-(12) encapsulate information both about a task itself and the environmental dynamics. Note that the spring model (12) does not only account for estimating the force $\hat{f}_{d}$ from $x$ in (12) but is also involved into the design of dynamics (11). We should also note that the goal $g^{\prime}$ is not a positional goal but involves both desired force and position information. By appropriately setting the parameters $\alpha_{z}, \beta_{z}$, $\gamma_{z}$ the system (11) is stable. The derivation of the set of stabilizing parameteres is straightforward through eigenvalue analysis and is not presented here due to space limitations. 


\section{B. Learning the nominal force representation}

Instead of having the goal information inside the system at each time step, we provide to the system only the final goal point and make it converge to this goal by following a desired route prescribed by the demonstrated force $f_{d}$. A similar approach has been shown in [3] to learn motion primitives by learning a control policy. In contrast to [3], we learn a demonstrated force pattern by learning the policy expressed by (11), (12). Therefore we modify equation (11) as follows $\tau \dot{x}=z-x-\gamma_{z} \int x+h \quad, \quad \tau \dot{z}=\alpha_{z}\left(\beta_{z}(g-x)-z\right)$,

where $h$ is a parametric nonlinear function, $\tau$ a time scaling constant, and $g$ the endpoint of the goal trajectory $g^{\prime}$. By learning $h$, we modify dynamics (15), (12) and make it evolve along the trajectory $f_{d}$. Function $h$ is defined as a weighted mixture of locally linear models as follows

$$
h=\frac{\sum_{i=1}^{N} w_{i} h_{i} v}{\sum_{i=1}^{N} w_{i}}
$$

where $w_{i}$ and $h_{i}$ are models' weights and output functions respectively. The weights $w_{i}$ are defined by Gaussian kernels with predefined centers and activation areas. The output functions $h_{i}$ are linear in the regression parameters and are trained by batch fitting in the input space represented by the phase space $v$. The phase variable $v$ evolves according to the following second order dynamical system

$$
\tau \dot{v}=\alpha_{v}\left(\beta_{v}(g-u)-v\right) \quad, \quad \tau \dot{u}=v
$$

where $\alpha_{v}$ and $\beta_{v}$ are set equal to $\alpha_{z}$ and $\beta_{z}$ respectively.

The learning process is as follows. Given demonstrated set of position $x_{d}$ and force $f_{d}$ data, we first compute the goal trajectory $g^{\prime}$ by (13) and then, the goal endpoint $g$. We substitute position $x$ in (15) by its demonstrated value $x_{d}$ and then we compute a desired trajectory for $h$ which is called $h_{d}$. In order to approximate the desired trajectory $h_{d}$, functions $h_{i}$ are trained by the locally weighted regression method. Given the learned $h, x$ is estimated from (15) and then, force $\hat{f}_{d}$ from (12), assuming initial conditions of $x, \dot{x}$ and $z$ equal to zero.

An important property of the proposed dynamical representation is the ability to scale the force profile of a task by modifying the goal $g$ of the system. This allows that a learned force profile can be easily scaled up or down for manipulation of stiffer or softer environments. By using a a phase variable $v$ instead of an explicit time variable, the system becomes autonomous which allows for easier online modication of its behavior.

\section{EXPERIMENTS}

\section{A. Experimental setup}

We evaluate our method in virtual manipulation tasks by using a 2 DOF linear-actuated haptic device shown in Fig. 3. Learning of interaction forces is demonstrated in a virtual environment which consists of a compliant object with planar surface and mass-spring-damper properties. The object is

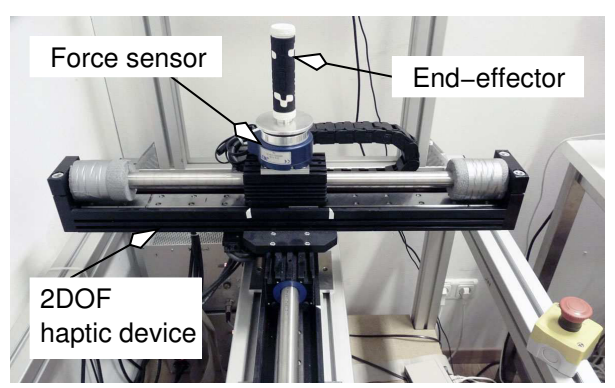

Fig. 3. Two degrees-of-freedom linear haptic device (ThrustTube).

placed such that its plane is always perpendicular to one direction of movement. Movement in the direction normal to the object's surface is, thus, constrained while movement in the other direction is unconstrained. The end-effector, which is the device's handle, is considered an inertia with a rendered inertia $m_{d}=40 \mathrm{~kg}$ and is assumed in continuous contact with the environment during the task. The reaction force from the environment is equal to the force exerted by the end-effector on it since slipping effects on the object's surface are not simulated.

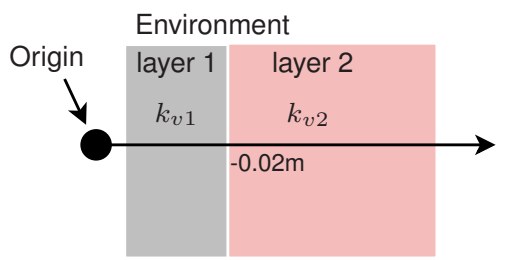

Fig. 4. Two-layer object where layers 1 and 2 have different stiffness coefficients $k_{v 1}$ and $k_{v 2}$. The user presses the object along the horizontal axis.

\section{B. Learning forces by demonstration}

A human user demonstrates the desired force for a manipulation task by moving the handle of the haptic device. During demonstration, we measure the demonstrated force $f_{d}$ exerted by the user and the demonstrated position $x_{d}$ of the handle. Both the user and the virtual environment exert a force on the handle and its movement is modulated accordingly. The reaction force in the constrained direction $f$ is rendered from interaction with the virtual object based on the following relationship $f=m_{v} \ddot{x}+d_{v} \dot{x}+k_{v} x$. The term $d_{v}$ represents the damping, $k_{v}$ the stiffness and $m_{v}$ the mass of the object. Demonstrated position $x_{d}$ and force $f_{d}$ data are employed for learning interaction forces by applying the methodology described in Section IV. We evaluate our method in two different types of tasks in homogeneous and inhomogeneous environments, respectively.

\section{Control implementation}

The haptic device is attached to a PC which runs the realtime linux operating system RTAI. The entire control scheme and also the virtual environment is implemented in MATLAB/Simulink from which realtime capable code is generated. The hardware device as well as the control loops run at a sampling rate of $1000 \mathrm{~Hz}$. The parallel force/position control law as given in (3), (4), and (5) is implemented. 


\section{Learning pressing skills}

First, we learn a pressing task for an object, which is required in grasping where appropriate force needs to be exerted onto the object to grasp and pick it up. The aim of this experiment is to demonstrate learning of interaction force in presence of environments with spatially varying stiffness. In particular we consider objects here with two layers with different stiffness coefficients $k_{v 1}$ and $k_{v 2}$ as illustrated in Fig. 4. The object has a planar surface (left boundary of layer 1) which is opposed perpendicularly to the horizontal direction of movement and lies at rest at $-0.001 \mathrm{~m}$ from the origin (black ball). The inner layer of the object lies at rest at $-0.02 \mathrm{~m}$ from the origin.

We learn appropriate forces to press these objects and reach their inner layer (layer 2) and the results are visualized in Fig. 5(a), (b). The point where the inner layer is reached is shown by a red marker on the position plot.

We assume one point of contact of the end-effector with the environment. We tested with two different objects. Task 1 is the pressing an object with outer stiffness $k_{v 1}=100 \mathrm{~N} / \mathrm{m}$ (grey layer) and inner stiffness $k_{v 2}=180 \mathrm{~N} / \mathrm{m}$ (pink layer). Task 2 is pressing an object with $k_{v 1}=500 \mathrm{~N} / \mathrm{m}$ and $k_{v 2}=$ $600 \mathrm{~N} / \mathrm{m}$. Both objects have damping of $d_{v}=50 \mathrm{Ns} / \mathrm{m}$ and mass $10 \mathrm{~kg}$.

Once forces are demonstrated, they are learned by the methodology described in Section IV. We observe that the proposed method successfully learns demonstrated interaction force in changing environments with approximation errors which do not exceed 4 percent of maximum demonstrated force. The approximation error is calculated by $\frac{\left|f_{d}-\hat{f}_{d}\right|}{f_{d \max }}$ where $f_{d_{\max }}$ is the maximum demonstrated force.

Learned force is executed via the control law (8) where the term $m_{d} \ddot{x}$ represents the total force command. Forces are successfully tracked by the controller while a perturbation in tracking is observed in both cases, when the second layer is reached which is characterized by a different stiffness value. The regions of perturbation are marked by red ellipses on the force plot in Fig. 5(b).

\section{E. Learning engraving skills}

In this experiment, the proposed method is tested to a simplified engrave-like task due to limited haptic rendering of object deformation effects. In engraving task 1 and task 2 , different environment has been used with stiffness 100 $\mathrm{N} / \mathrm{m}$ and $280 \mathrm{~N} / \mathrm{m}$ respectively. Both has damping $50 \mathrm{Ns} / \mathrm{m}$ and mass $10 \mathrm{Kg}$. Objects' interface is planar, opposed perpendicularly to the horizontal direction of movement, and lies at rest at distance $-0.001 \mathrm{~m}$ from the origin.

We learn demonstrated force for engraving on these two environments and the results are visualized in Fig. 6(a),(b). Desired force is demonstrated by moving from the origin, in the horizontal direction, and pressing with random forces the stiff and compliant objects. We observe that successful learning of interaction forces takes place with approximation errors which do not exceed 6 percent of maximum demonstrated force.
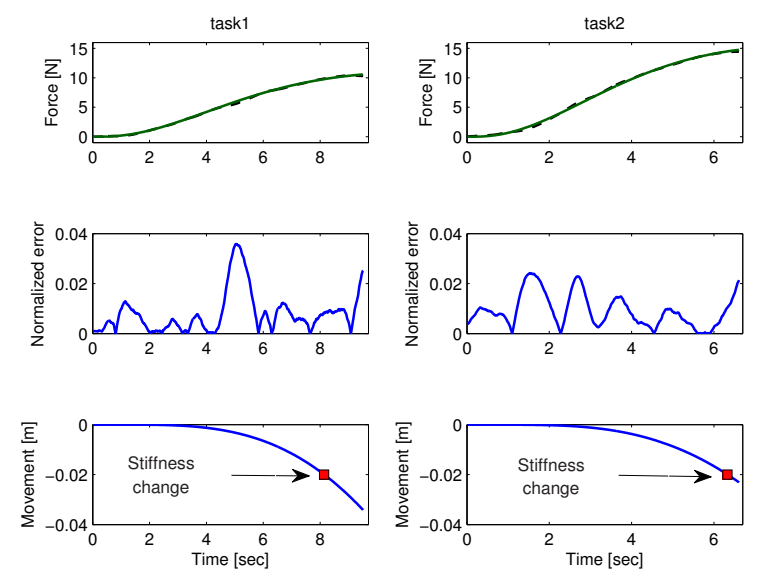

(a)
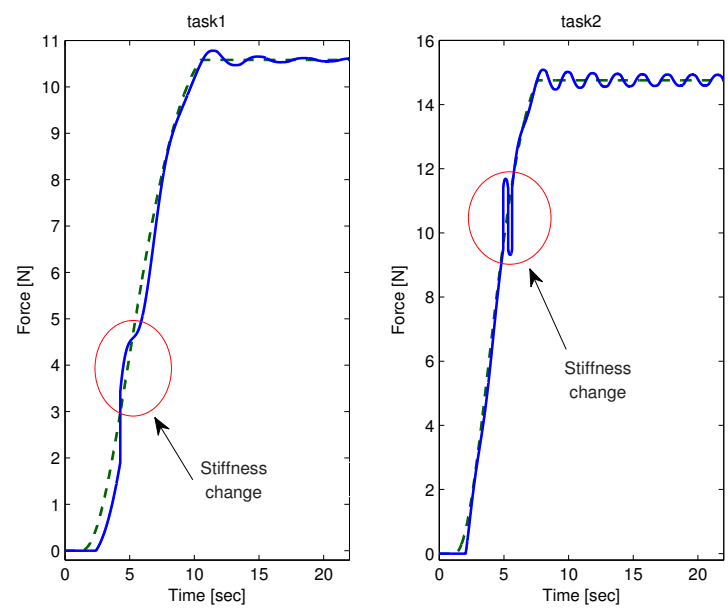

(b)

Fig. 5. Learning and execution result of pressing skills. (a) (top row) demonstrated (dashed black) and learned (solid green) force, (middle) approximation error, (bottom) demonstrated constrained movement. (b) learned (dashed green) and measured (solid blue) force during the execution.

We employ learned engraving skills shown in Fig. 7 to perform on two other objects with same geometrical configuration but different physical properties. The new objects have same damping and mass with the previous ones while their stiffness is $200 \mathrm{~N} / \mathrm{m}$ for the first and $300 \mathrm{~N} / \mathrm{m}$ for the second object. The user exerts a force starting from the origin to manipulate the objects which are placed at $-0.001 \mathrm{~m}$ from the origin. To engrave the first object, we combine in series learned forces of tasks 1 and 2 shown in Fig. 6(a) and execute for 8 and $9 \mathrm{sec}$. respectively. To manipulate the second stiffer object, we scale up the force signals by setting the goal $g$ in (15) 1.5 times the original goal and scaled forces are executed in series for 8 and 9 sec. respectively. Force scaling is illustrated in Fig. 7 (a1), (a2). Force skills are executed via (8). In Fig. 7 (c), (d), we can observe how objects' dimension along the constrained direction of movement deforms over time following exertion of learned and scaled forces respectively. We observe that by scaling up the force in the case of the stiffer environment (Fig. 7 (d)), 

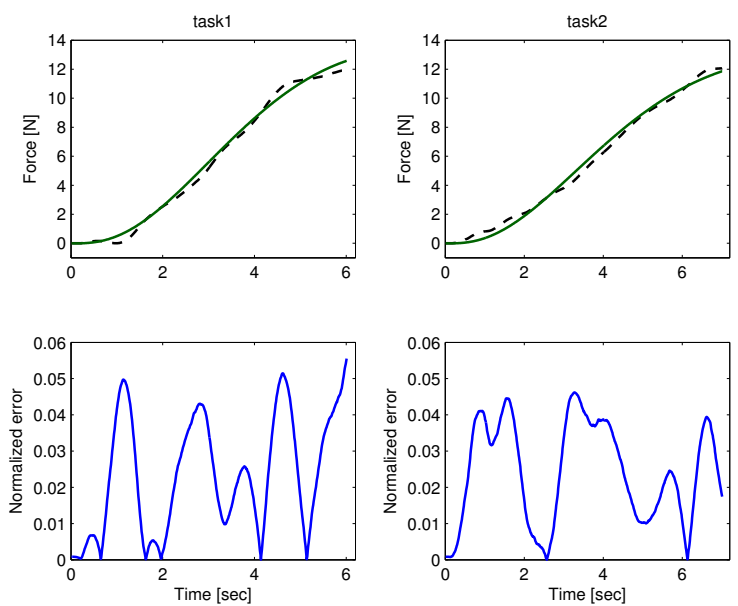

(a)
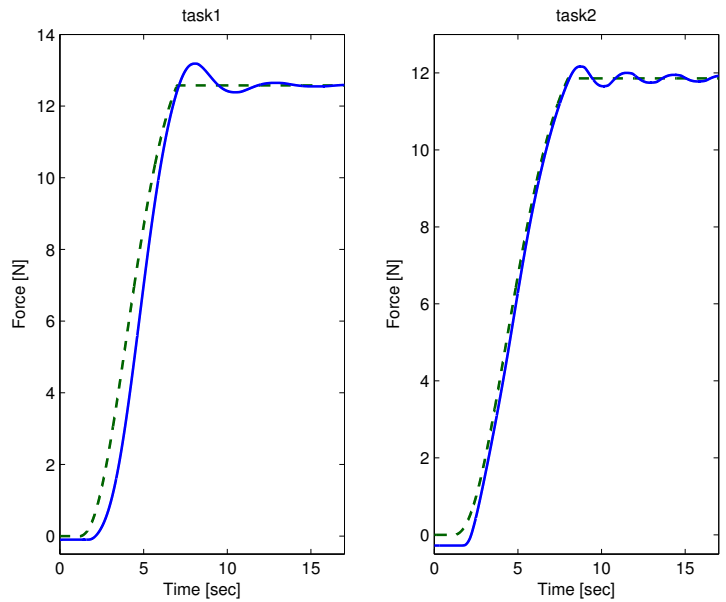

(b)

Fig. 6. Learning and execution result of engraving skills. (a) (top) demonstrated (dashed black) and learned (solid green) force, (bottom) approximation error. (b) learned (dashed green) and measured (solid blue) force during the execution.

resulting constrained movement becomes the same with that in the softer material (Fig. 7 (c)).

\section{CONCLUSIONS AND FUTURE WORKS}

In the present study, we propose a method to learn interaction force skills in presence of compliant external dynamics from human demonstrations. An interaction force is encoded in terms of a parameterized time-invariant differential equation based upon the parallel force/position control law. The parameters of this equation are trained by locally weighted regression to learn an interaction force pattern. The proposed method is applied into two tasks in virtual environments: pressing of inhomogeneous objects and simple engraving tasks in different environments.

Future work will focus on extending the current framework to allow for generalization of force skills in real-time by employing information about the environmental state. In addition, self-improvement of force skills will be investigated by using coupling dynamics and reinforcement learning.
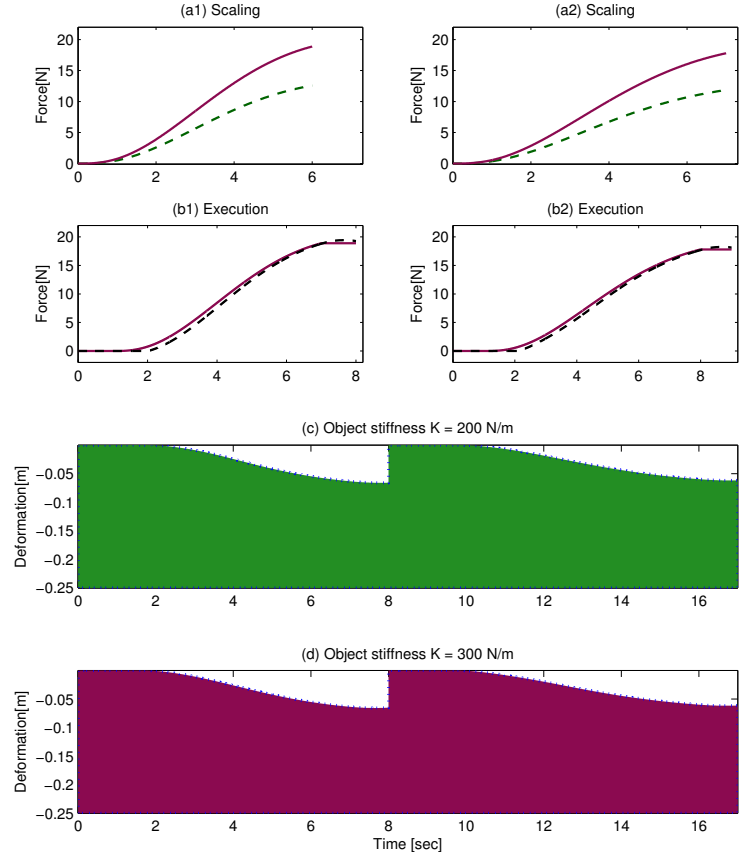

Fig. 7. Engraving using learned force in tasks 1 and 2 in Fig. 6(a). (a1) (a2) Learned force (dashed green) and scaled force (solid red). (b1), (b2) Execution of scaled forces for (b1) 8 sec. and (b2) 9 sec., scaled (solid red) and measured (dashed black) force. Object's dimension over time along the constrained direction of movement by executing (c) learned forces and (d) scaled forces.

\section{ACKNOWLEDGMENTS}

This research is partly supported by the DFG excellence initiative research cluster "Cognition for Technical Systems CoTeSys" and the Bavarian Elite Aid Act (BayEFG)-Elite Network of Bavaria.

\section{REFERENCES}

[1] S. Chiaverini and L. Sciavicco, The parallel approach to force/position control of robotic manipulators, IEEE Trans. Robot. Automat., vol. 9, 1993, pp 361-373.

[2] F. Caccavale, C. Natale, B. Siciliano and L. Villani, Resolvedacceleration control of robot manipulators: A critical review with experiments, Robotica, vol. 16, 1998, pp 565-573.

[3] A. Ijspeert, J. Nakanishi and S. Schaal, Learning attractor landscapes for learning motor primitives, in Advances in Neural Information Processing Systems, MIT Press, 2003, pp 1523-1530.

[4] N. Hogan, Impedance control: An approach to manipulation: Part I, Part II, Part III., ASME J. Dynam. Syst., Meas. Contr., vol. 107, 1985, pp 1-24.

[5] M. Kawato, Internal models for motor control and trajectory planning, Current Opinion in Neurobiology, vol. 9, 1999, pp 718-727.

[6] S. Calinon, F. Guenter and A. Billard, On learning, representing and generalizing a task in a humanoid robot, IEEE transactions on systems, man and cybernetics, Part B. Special issue on robot learning by observation, demonstration and imitation, vol. 37, 2007, pp 286-298.

[7] A. J. Ijspeert, J. Nakanishi and S. Schaal, Movement imitation with nonlinear dynamical systems in humanoid robots, Proceedings of the IEEE International Conference on Robotics and Automation (ICRA2002), 2002, pp 1398-1403.

[8] Dongheui Lee, Christian Ott, Yoshihiko Nakamura, Mimetic Communication Model with Compliant Physical Contact in Human-Humanoid Interaction, The International Journal of Robotics Research, 29, no. 13, 2010, 1684-1704.

[9] L. Sciavicco and B. Siciliano, Modeling and Control of Robot Manipulators, Second edition, Springer-Verlag New York, Inc.,Secaucus, NJ, 2000.

[10] O. Khatib, A unified approach for motion and force control of robot manipulators: the operational space formulation, IEEE J. Robotics and Automation, vol. RA-3, 1987, pp 43-53. 